\title{
INTERFERÊNCIAS DE BARRAMENTOS NO REGIME HIDROLÓGICO DO ESTUÁRIO DO RIO CATÚ-CEARÁ-NORDESTE DO BRASIL
}

\section{Barrier Interference on Catú Estuarine Hydrologic System - Ceará - NE - Brazil}

Lidriana de Souza Pinheiro

Professora Dra. da Universidade Federal do Ceará

Bolsista PQ-CNPq

Fortaleza/CE - Brasil

lidriana@ufc.br

Jáder Onofre de Morais

Professor Titular da Universidade Estadual do Ceará

Bolsista PQ-CNPq

Fortaleza/CE - Brasil

jaderonofre@gmail.com

Artigo recebido para publicação em 05/10/2009 e aceito para publicação em 05/04/2010

RESUMO: $\quad$ O estuário do rio Catú localiza-se a 26 km a sudeste de Fortaleza, no Município de Aquiraz. O objetivo desse estudo foi avaliar os impactos de barramentos de pequeno e médio porte no aporte de água doce e sua repercussão na evolução morfológica e fragilidade ambiental do referido sistema. Foram escolhidas e analisadas as principais intervenções no escoamento superficial, formas de uso e ocupação das margens no período entre 1990 e 2004. O impacto dessas intervenções na vazão fluvial, com foco nos barramentos, foi simulado com uso de modelos e equações semi-empíricas, em função da ausência de estações de monitoramento fluviométrico. A construção do Açude Público Catú-Cinzento, no alto curso, diminuiu em 30\% a contribuição de águas para a lagoa do Catú e consequentemente para o estuário. Isto resultou na diminuição do volume estuarino de $8.507 \mathrm{~m}^{3}$ para $2.045 \mathrm{~m}^{3}$ sob as mesmas condições climáticas e de marés. O tempo de residência das águas é de aproximadamente 18 dias, com baixa capacidade depuradora. Os processos de sedimentação ali existentes configuram o ambiente em causa como estuarino-lagunar em fase evolutiva para ambiente lacustre.

Palavras-chave: Estuário. Barragens. Hidrologia. Tempo de residência. Morfologia.

ABSTRACT: The Catú River estuary lies $26 \mathrm{~km}$ SW of Fortaleza in Aquiraz municipality. This study aims to evaluate the small and medium barrier impacts in fresh water inputs and effects on the morphological evolution and environmental fragility of the estuary system. The main superficial flow interventions have been chosen and analyzed as well as the margins use and occupation from 1990 to 2004. The impacts focused in the barrier spilling were simulated through semi-empirical equation models, once there was no fluviometry monitoring stations. The over dimension of the Catú river spill wall, in 993, gave rise to flow relentless in rainy seasons for volumes less than $1 \mathrm{~m}^{3} . \mathrm{s}^{-1}$ favoring the mouth silting, estuary area shortage and mangrove decreasing. Afterwards, the wall resizing increased flow rate to $7 \mathrm{~m}^{3} . \mathrm{s}^{-1}$ due to flash flood in upstream areas at rainy season, allowing channel clearing and water oxygenation. The public dam Catú-Cinzento, in the river backland, decreases in 30\% the lake water input and in the estuary. This caused the estuary water volume shortage from $8.507 \mathrm{~m}^{3}$ to $2.045 \mathrm{~m}^{3}$ under the same climatic 
and tide conditions. The water residence time is 18 days with low depuration capacity. The existing sedimentary processes configure the study environment as lagoon-estuary evolving to lake.

Keywords: Estuary. Barrier. Hydrology. Residence time. Morphology.

\section{INTRODUÇÃO}

Os rios da região semi-árida do Nordeste brasileiro, onde está inserida a área estudada, são intermitentes, fluindo somente durante a estação chuvosa. A penetração da água do mar nos vales durante as marés altas impede que esses rios fiquem sem a comunicação com o oceano durante a estiagem. Nesta estação, a vazão é praticamente nula e consequentemente não há uma gradativa diluição da água do mar pela água do rio. As espécies nativas nessas regiões sobrevivem, distribuem-se e reproduzem-se sob influência das grandes variações sazonais de vazões (TOWNSEND; HILDREW, 1994; STANFORD 1996).

Esses estuários podem ser considerados como temporários, com características de circulação e mistura tipicamente estuarinas (DYER, 1997; MIRANDA et al., 2002), restritas ao período chuvoso. A entrada de água doce nesse período tem consequências físicas, químicas e biológicas importantes (ALBER, 2002; SKLAR; BROWDER, 1998).

Os efeitos positivos desta afluência são observados nos padrões de circulação estuarina, na formação de gradiente de salinidade, transporte de sedimentos, fornecimento de nutrientes, produção de pescado (GENZ; LESSA, 2008) e controle do assoreamento e erosão da foz (PINHEIRO et al., 2006).

Como produto das condições climáticas mais severas, nas regiões tropicais semi-áridas, a velocidade dos ventos é mais intensa, juntamente com o transporte e disponibilidade de sedimentos, fazendo com que o domínio das formações arenosas, sob a forma de dunas, contribua significativamente para a obstrução das reentrâncias costeiras (MORAIS et al., 2002). Em consequência, as drenagens de pequeno porte evoluem para sistemas fluvio-lagunares ou estuarino-lagunares (KJERFVE, 1994; MARTIN; DOMINGUEZ, 1994), apresentando em alguns casos, condições de hipersalinização, ou seja, salinidade superiores a 50 usp (KJERFVE et al., 1996). Neste caso, a função de espigão hidráulico no período chuvoso (DOMINGUEZ et al., 1983) é fundamental para reverter à obstrução de canais favorecendo a circulação estuarina e os processos de sedimentação.

Além das adversidades climáticas, a interferência da hidrologia estuarina pela construção de barragens compromete significativamente a sustentabilidade ambiental desses ecossistemas.

Barramentos implicarão no avanço da intrusão salina, aumento do tempo de residência dos estuários, hipersalinização e na redução da carga de sedimentos e nutrientes para a zona Costeira (WOLANSKI et al., 1996; ALBER, 2002; MARINS et al., 2003; KITHEKA et al., 2004, ARAÚJO et al., 2006; MOLISSANI et al., 2006; PINHEIRO et al., 2006; GENZ; LESSA, 2008). Consequentemente problemas socioeconômicos nas comunidades que exploram os recursos naturais ali existentes serão intensificados.

Estes processos e impactos são observados no Rio Catú, que nasce no Município de Horizonte e escoa por aproximadamente $30 \mathrm{~km}$ até desaguar no distrito de Prainha de Aquiraz. A sua origem, configuração atual e fragilidade ambiental estão associados a morfodinâmica de dunas no Holoceno (MORAIS et al., 2002) e a redução de vazão hídrica pela construção do vertedouro da Lagoa do Catú e do Açude Público Catú-Cinzento na última década.

No Brasil, estudos hidrogeomorfológicos envolvendo análises de vazões em rios regularizados ou não são cada vez mais realizados com a utilização de modelos e base de dados de agências e/ou instituições de monitoramento (FERNANDEZ; SOUZA FILHO, 1995; LIMA; ZAKI, 1998; CARVALHO; CUNHA 1998; CAVALCANTE, 2001; BRANDT, 2002; LATRUBESSE et al., 2005; ARAÚJO et.al., 2006). Entretanto, dados hidrológicos são de difíceis aquisições 
para inúmeras áreas, especialmente no semi-árido, em razão da carência de estações fluviométricas para monitoramento. Em geral, a Agência Nacional de Águas - ANA dispõe de dados de vazão, pelo estabelecimento de curvas chaves, para os principais sistemas hidrológicos do país.

Deste modo, nos estudos envolvendo sistemas hidrológicos menores e com ausência de séries temporais de dados monitorados é necessário o uso de equações empíricas ou semi-empíricas para entender o comportamento hidrológico no estuário.

Por isso, o objetivo deste estudo foi avaliar os impactos dos barramentos no regime hidrológico do estuário do rio Catú e suas possíveis repercussões na evolução morfológica e fragilidade ambiental.

\section{LOCALIZAÇÃo DA ÁREA}

O estuário do rio Catú localiza-se a $26 \mathrm{~km}$ de Fortaleza, no distrito de Prainha no Município de Aquiraz. O acesso se dá pela rodovia CE-040. Na área em questão, além dos impactos de ordem natural, decorrentes do barramento do Rio Catú pelo campo de dunas móveis, foi construída uma barragem de pequeno porte, localmente denominada de passagem molhada, que reduziu significamente o fluxo das águas para o sistema.

Na década de 1990 foi feito um vertedouro para minimizar os problemas de inundações na planície lacustre formada pelo barramento anterior (GOMES, 2003). O somatório desses processos causou a diminuição da área estuarina, prejuízos sobre a qualidade de suas águas e perda de atrativos para o lazer devido as mudanças bruscas dos parâmetros físico-químicos e o incremento do assoreamento de todo o sistema. Aliase a isso, os danos causados à biota e à comunidade local pela redução da diversidade biológica e deterioração dos recursos naturais (MORAIS et.al., 2002).

A evolução do estuário do Catú está diretamente relacionada à morfodinâmica dos campos de dunas móveis ao longo do Quaternário. Essas dunas impulsionadas pelos ventos de E-SE migraram na direção do canal estuarino dando origem a Lagoa do Catú e restringindo a ação das marés ao primeiro setor. Aliado a este processo, a dinâmica da foz passou a ser controlada pela ação da deriva litorânea tendo em vis- ta que a função de espigão hidráulico da drenagem ficou limitada e/ou ausentes. Isso resultou na formação de cordões arenosos que lentamente barraram a foz do rio. No período chuvoso, a barra era reaberta e criavase um ambiente sazonal com características estuarinas (MORAIS et al.; 2002).

Em 1993, a Prefeitura de Aquiraz realizou uma intervenção no barramento natural da lagoa na tentativa de aumentar o armazenamento de água e garantir o abastecimento do município que estava comprometido pelo longo período de estiagem dos anos antecedentes. No ano seguinte, as precipitações situaram-se acima da média, resultando em inundações das planícies aluviais à montante da estrutura e colocando em risco as urbanizações e atividades agro-industriais ali existentes (GOMES, 2003). Á jusante, os impactos no estuário foram imediatos, com o aceleramento do fechamento da foz pela ação da deriva litorânea e deflação eólica.

Em 1995, através de estudos solicitados pela Secretaria de Recursos Hídricos do Estado do Ceará foi sugerida a construção de um vertedouro na área de barramento natural das dunas com rebaixamento da cota de sangria de $8.84 \mathrm{~m}$ para $5.84 \mathrm{~m}$, com o intuito de regularizar as vazões e evitar, dessa forma, as inundações e impactos a fauna e flora do estuário. A vazão regularizada afluente ao estuário foi projetada para $0,18 \mathrm{~m}^{3} \cdot \mathrm{s}^{-1}$ (FIGURA 2). Neste período foi construída uma barragem de terra no início do terço superior da bacia do Rio Catú, nas localidades de Cinzenta e Guarda (SRH, 1995).

No ano de 2003 foi concluída a barragem CatúCinzento com capacidade de $27,1 \times 10^{6} \mathrm{~m}^{3}$ e vazão regularizada de $0,2 \mathrm{~m}^{3} \cdot \mathrm{s}^{-1}$. Na região estuarina e faixa de praia, a ocupação das margens ultrapassou o percentual de $85 \%$. A diminuição do espigão hidráulico e o contínuo aporte de areia promoveram a migração da drenagem de Leste para Oeste.

$\mathrm{O}$ aproveitamento das águas fluviais, com o fechamento de um rio para a formação do reservatório, assim como o aproveitamento da planície de inundação, através de obras de canalização, está associado à geração de uma série de alterações fluviais, com repercussões na zona costeira. A construção de barragens em vales fluviais, sejam elas através de processos naturais ou antrópicos, rompe a sequência natural 


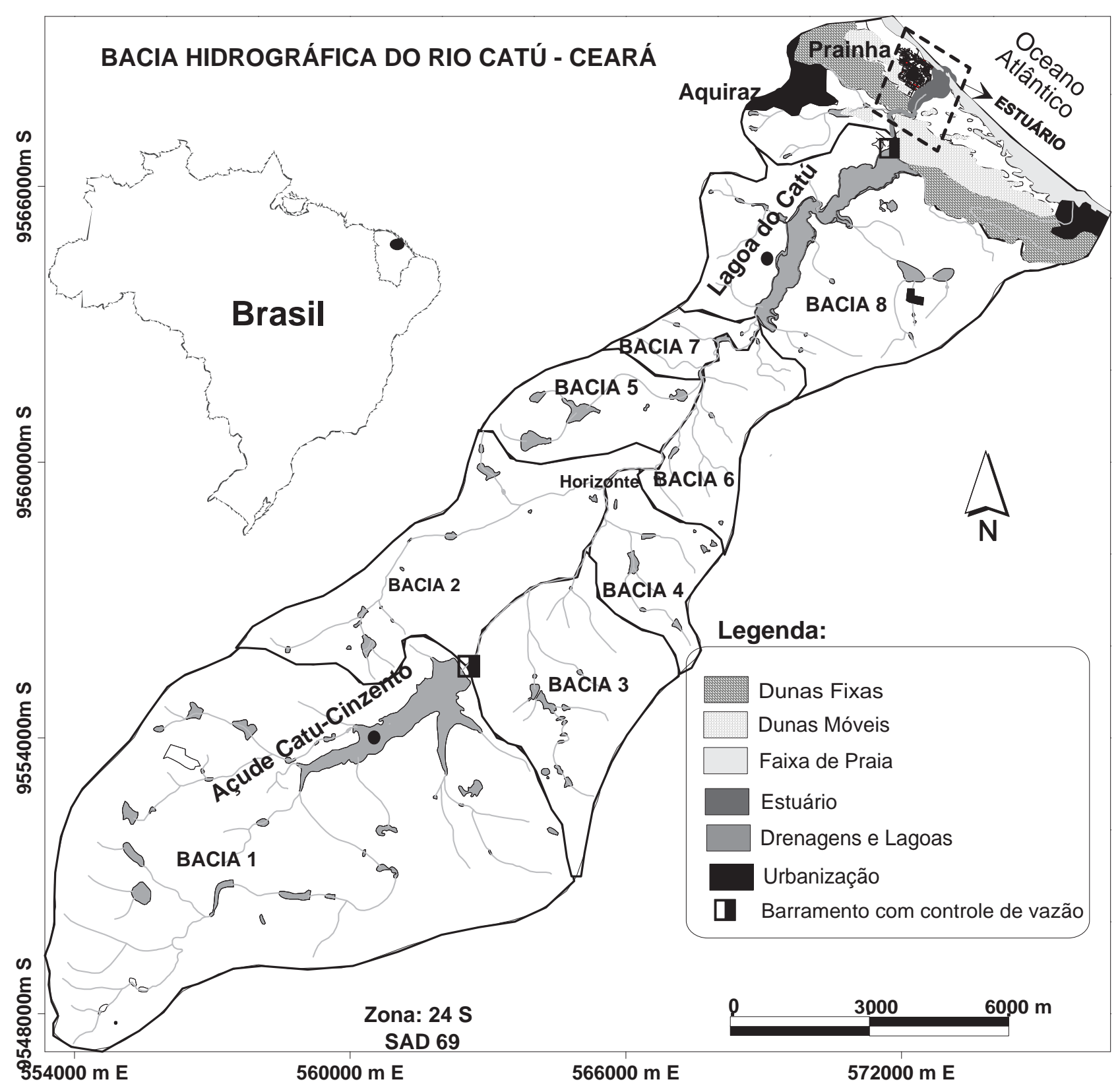

FIGURA 1: Bacia hidrográfica do rio Catú com indicação dos barramentos de pequeno e médio porte e áreas com controle de vazões ([

dos rios em três áreas distintas: Na parte a montante da barragem, o nível de base local é levantando, alterando a forma do canal e a capacidade de transporte sólido.

Isso ocasiona o aumento de sedimentos no reservatório, modificando o seu tempo útil, mudanças na qualidade da água armazenada e na biota local. $\mathrm{Na}$ parte jusante do reservatório, o regime do rio sofre modificações, devido ao controle artificial das descargas líquidas e de sedimentos. Com isso acarreta o aprofundamento do rio podendo causar significativas mudanças na foz.

Os estudos existentes na bacia hidrográfica do Catu estão restritos ao sistema lacustre, cuja influência da maré é nula. Dentre eles destacam-se os de Gomes (2003) e os boletins de monitoramento da qualidade 


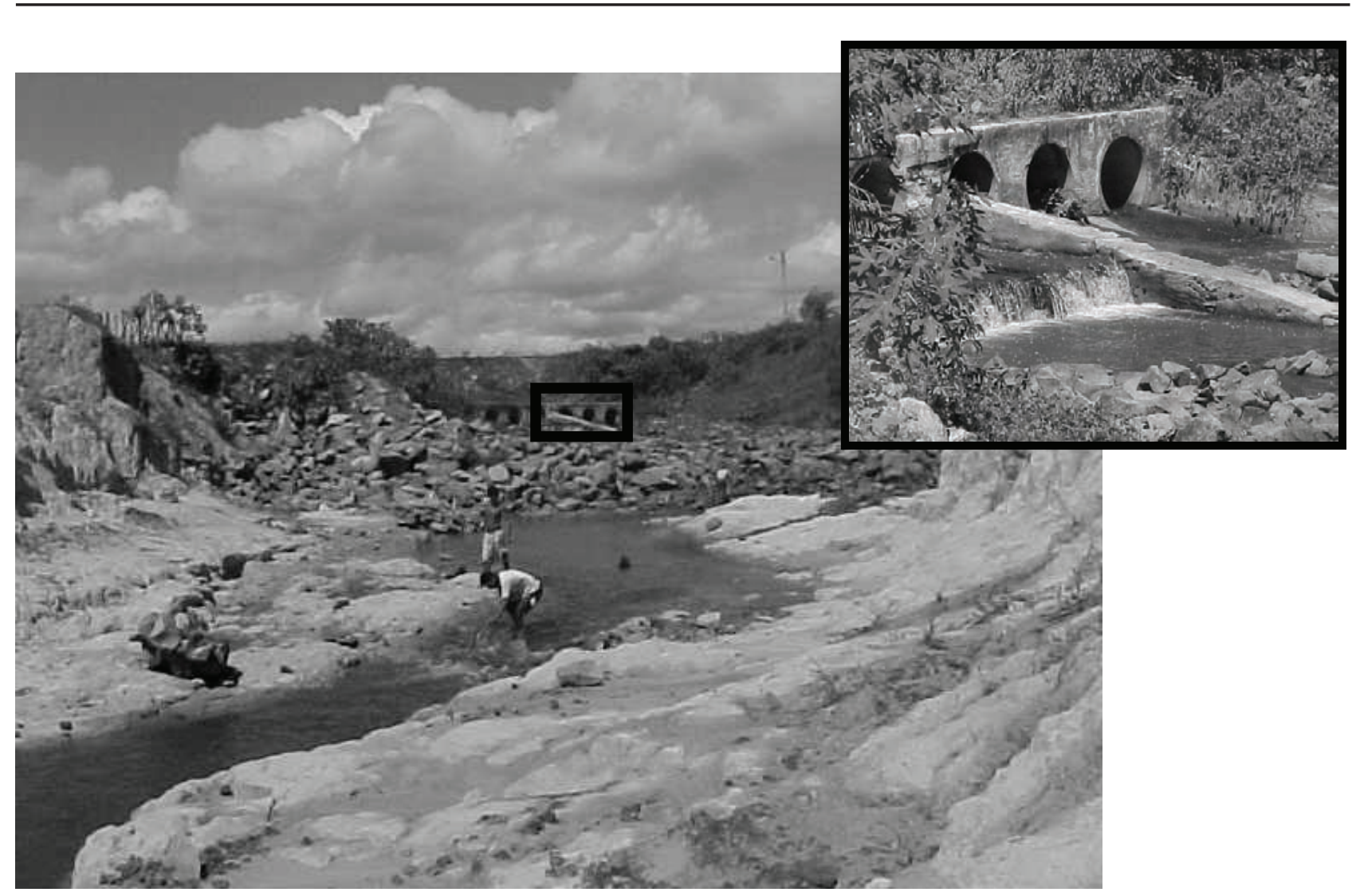

FIGURA 2: Vertedouro construído em 2003 para regularização do volume da Lagoa do Catú para fins de abastecimento e controle de inundações.
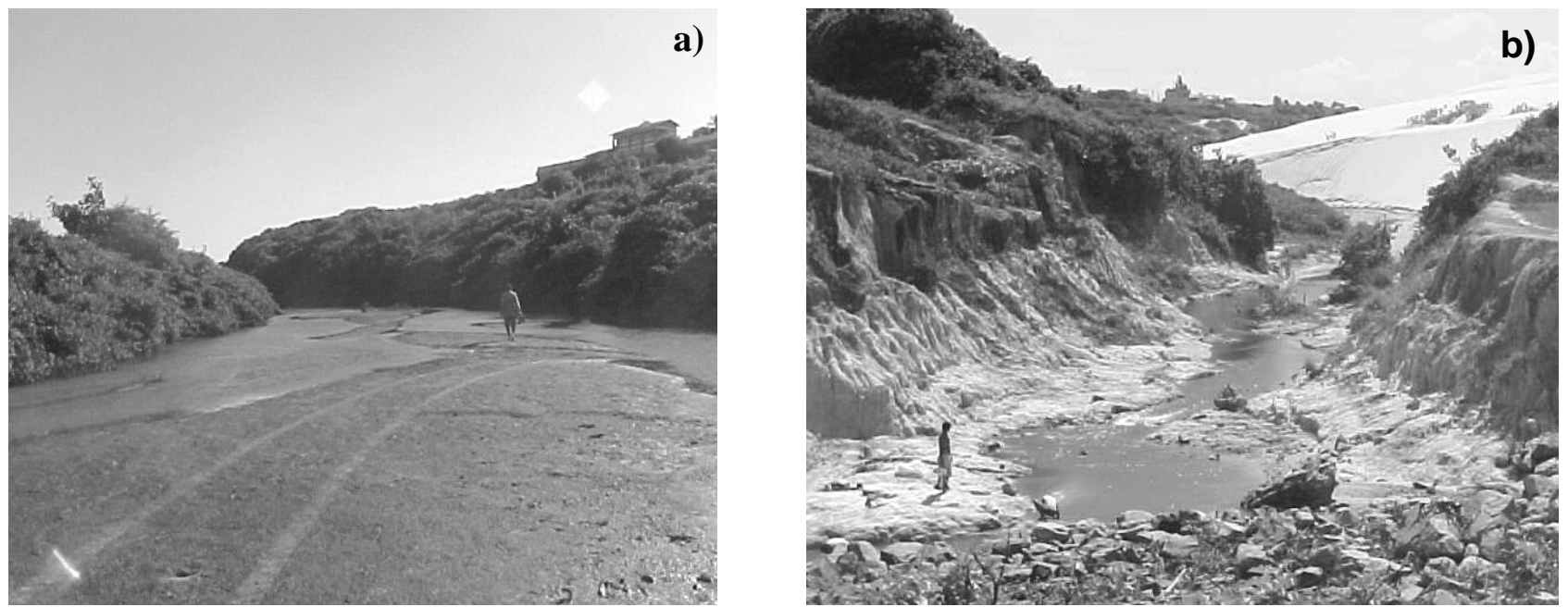

FIGURA 3: (a) No período de estiagem do ano de 2003, a vazão hidráulica controlada pelo vertedouro da lagoa do Catú não possibilita a comunicação das águas continentais com o estuário. (b) Diminuição da vazão do Rio Catú pelo represamento das águas no período chuvoso de 2004. Ao longo do percurso a drenagem é barrada parcialmente pelos campos de dunas móveis. 
da água para abastecimentos realizados pela Companhia de Água e Esgoto do Ceará-CAGECE. Mesmo o estuário sendo uma área à jusante desta lagoa, os dados do monitoramento não foram extrapolados. Isto foi devido o escoamento ser próximo de zero no período de estiagem, impossibilitando a comunicação das águas lacustres e o estuário (FIGURA 3-a). O resultado disto é a estagnação de águas com aumento da salinidade pelo processo de evaporação. No período chuvoso do ano de 2004, apesar das médias pluviométricas terem sido superiores as médias da região, a liberação de água no vertedouro da Lagoa do Catú foi muito reduzida (FIGURA 3-b).

\section{METODOLOGIA}

\section{Arcabouço evolutivo}

A bacia de drenagem é a origem do sistema de rios que suprirá o estuário de água fluvial, sedimentos, substâncias orgânicas e inorgânicas e eventualmente poluentes (MIRANDA et al., 2002). Assim, é importante levar em consideração as alterações ocorridas em toda a bacia de drenagem, para que se possa ter uma noção precisa da magnitude dos processos que atuam nas áreas estuarinas. Por isso, a primeira etapa desse estudo consistiu no levantamento das principais transformações na bacia de drenagem desde o início da década de 1990 que repercutissem nos processos estuarinos. Foram utilizadas informações disponibilizadas na literatura científica, banco de dados das Prefeituras Municipais e órgãos municipais e estaduais de gerenciamento dos recursos hídricos. Processos morfodinâmicos atuais, tais como migração de dunas e de barras arenosas na desembocadura, também foram considerados.

\section{Compartimentação da Bacia Hidrográfica}

A descarga fluvial é um dos principais fatores que controlam a hidrodinâmica de estuários. As variações sazonais no aporte de água doce afetam diretamente os gradientes longitudinais de salinidade e assim, os processos de circulação e mistura. Em se tratando de bacias hidrográficas muito grandes, a descarga fluvial tende a tornar-se espacialmente heterogênea, apresentando áreas com maior ou menor disponibilidade hídrica.

Para melhorar a acuidade das estimativas de descarga fluvial utilizadas nesse estudo, a bacia de

TABELA 1: Bacias e Área de contribuição do Reservatório Catú-Cinzento e do Estuário do Rio Catú.

\begin{tabular}{|c|c|c|c|}
\hline Bacias de Contribuição & Área $\left(\mathrm{km}^{2}\right)$ & Reservatórios $\left(\mathrm{km}^{2}\right)$ & Total $\left(\mathrm{km}^{2}\right)$ \\
\hline \multicolumn{4}{|c|}{ Reservatório Catú-Cinzento } \\
\hline Bacia 1 & 65 & 3,8 & 61,20 \\
\hline \multicolumn{4}{|c|}{ Lagoa do Catú } \\
\hline Bacia 2 & 19,40 & 0,30 & 19,10 \\
\hline Bacia 3 & 14,80 & 0,30 & 14,50 \\
\hline Bacia 4 & 6,80 & 0,20 & 6,60 \\
\hline Bacia 5 & 7,80 & 0,40 & 7,40 \\
\hline Bacia 6 & 7,40 & 0,07 & 7,30 \\
\hline Bacia 7 & 2,90 & 0,01 & 2,90 \\
\hline Bacia 8 & 25,60 & 2,90 & 22,70 \\
\hline \multicolumn{4}{|c|}{ Estuário do Rio Catú } \\
\hline Bacia $9 * *$ & 4,70 & 1,79 & 2,90 \\
\hline Bacia 10 & 3,50 & 0,00 & 3,50 \\
\hline Bacia 11 & 9,08 & 0,00 & 9,10 \\
\hline Estuário & 0,18 & 0,00 & 0,20 \\
\hline Total & 167,00 & 10,00 & 157,00 \\
\hline
\end{tabular}

**Sistema totalmente barrado com vazão afluente ao estuário do Rio Catú igual a $0 \mathrm{~m}^{3} \cdot \mathrm{s}^{-1}$. 
drenagem do Rio Catú foi dividida em onze subbacias, a partir do modelo digital do terreno (MDT), dentre as quais foi incluída a bacia afluente ao reservatório Catú Cinzento (TABELA 1). O MDT foi gerado a partir de interpolação kriging dos dados extraídos das cartas planialtimétricas gentilmente cedidas pela Secretaria Estadual dos Recursos Hídricos (SRH). A partir disto foram calculadas as áreas das sub-bacias e dos reservatórios.

$\mathrm{O}$ arranjamento espacial das áreas de contribuição hídrica e dos reservatórios com vazões regularizadas e monitoradas pela Companhia de Gerenciamento dos Recursos Hídricos do Estado do Ceará-COGERH estão detalhadas na FIGURA 1.

\section{Modelos Hidrológicos}

A estimativa da descarga fluvial da bacia de drenagem para o sistema estuarino $\left(Q_{f}\right)$ foi realizada considerando-se duas hipóteses: ausência e presença do barramento. Devido ao fato de que não há monitoramento e estações fluviométricas na bacia estudada foram utilizadas equações semi-empíricas para estimar a contribuição da bacia de drenagem para o sistema estuarino. Esse método foi aplicado satisfatoriamente por Medeiros e Kjerfve (1993), Kjerfve et al. (1996), Pinheiro (2003), Dias (2005) e Molisani et al. (2007) em estudos hidrológicos de sistemas estuarinos de Itamaracá (PE), Aruarama (RJ), Malcozinhado (CE), Timonha (CE) e Cocó (CE).

O modelo climático utilizado para estimativa do escoamento superficial foi proposto por Kjerfve (1990), baseado em dados de precipitação e temperatura representativos para cada sub-bacia. As séries históricas das precipitações e temperaturas do ar dos últimos trinta anos na Bacia de Drenagem do Rio Catú foram cedidas pala Fundação Cearense de Meteorologia e Recursos Hídricos (FUNCEME). Detalhamentos diários das precipitações para simular o impacto imediato após construção do Açude CatúCizento foram obtidos no site da FUNCEME (http/ www.funceme.br).

De acordo com o modelo hidrológico, a vazão de água doce de cada sub-bacia $\left(\mathrm{q}_{\mathrm{f}}\right)$ em $\mathrm{m}^{3} \mathrm{~s}^{-1}$ é dada por:

$$
q_{f i}=a_{i} \times P_{\times} \frac{\Delta f}{P}
$$

(Equação 1)

onde, $\mathrm{a}_{\mathrm{i}}$ é a área superficial da sub-bacia $\mathrm{em}^{2}$, P representa a contribuição pela precipitação pluviométrica em $\mathrm{m} \cdot \mathrm{s}^{-1} \mathrm{e}(\Delta \mathrm{f} / \mathrm{P})$ a razão de escoamento superficial. De acordo com Schreiber (1904) apud Miranda et al. (2002), o valor adimensional da razão de escoamento superficial pode ser calculado através da seguinte equação:

$$
\frac{\Delta f}{P} \approx \exp \left(\frac{-E_{v}}{P}\right)
$$

Em que $\mathrm{E}_{\mathrm{v}}$ é a taxa média anual de evapotranspiração potencial em $\mathrm{m} \mathrm{s}^{-1}$, obtida em função da temperatura de acordo com a equação proposta por Holland (1978) apud Miranda et al. (2002):

$E_{v}=0,3805_{x} \exp \left(\frac{-4,62 \times 10^{3}}{T}\right)$

(Equação 3)

Nessa equação a temperatura deve ser expressa em Kelvin (K) e $\mathrm{E}_{\mathrm{v}}$ é dada em $\mathrm{m} \cdot \mathrm{s}^{-1}$ tendo resultados satisfatórios do Equador até a latitude de $70^{\circ}$ (Norte e Sul). O valor de $\mathrm{E}_{\mathrm{v}}$ somente é significativo quando são utilizados dados médios de temperatura de longos períodos de tempo (KJERFVE, 1990).

$\Delta \mathrm{f}$, por sua vez, pode ser obtida, em $\mathrm{m} \cdot \mathrm{s}^{-1}$, através da relação:

$$
\Delta f=\frac{Q_{T}}{A_{E}}
$$

(Equação 4)

Então, a contribuição da bacia de drenagem para o sistema estuarino através do escoamento superficial $\left(Q_{f}\right)$ em $m^{3} \cdot s^{-1}$ é dada por:

$$
Q_{f}=\sum_{i=1}^{i=n} q_{f i}
$$

No sistema estuarino, a contribuição de água doce pelo escoamento difuso $\left(\mathrm{Q}_{\mathrm{d}}\right)$ foi obtida através da seguinte equação:

$$
Q_{d}=A_{E} \times(P-E)
$$

(Equação 6) 
Onde $A_{E}$ é a área da bacia estuarina em $\mathrm{m}^{2}$ e $Q_{d}$ é dado em $\mathrm{m}^{3} \mathrm{~s}^{-1}$. As taxas de precipitação e evaporação devem ser aplicadas em $\mathrm{m} \cdot \mathrm{s}^{-1}$.O aporte total de água doce que abastece o sistema estuarino $\left(\mathrm{Q}_{\mathrm{T}}\right) \mathrm{em}$ $\mathrm{m}^{3}$. $\mathrm{s}^{-1}$ é:

$$
Q_{T}=Q_{f}+Q_{d}
$$

(Equação 7)

O balanço de água é um dos principais processos que condicionam a hidrodinâmica de estuários. $\mathrm{O}$ volume de água que entra e sai da bacia estuarina é dado pela seguinte equação:

$$
\frac{\Delta \mathrm{V}}{\Delta \mathrm{t}}=\mathrm{Q}_{f}+\mathrm{Q}_{\mathrm{p}}+\mathrm{Q}_{\mathrm{E}}+\mathrm{Q}_{\mathrm{as}}+\mathrm{Q}_{\mathrm{r}}+\mathrm{Q}_{\mathrm{c}}+\mathrm{Q}_{0}
$$

(Equação 8)

Essa equação expressa a variação de volume do estuário $(\Delta \mathrm{V})$ de acordo com os fluxos de entrada $\mathrm{e}$ saída de água no sistema em um espaço de tempo $\Delta$ t. $Q_{f}$ representa a descarga de água doce que escoa da bacia de drenagem para o estuário; $\mathrm{Q}_{\mathrm{P}}$ e $\mathrm{Q}_{\mathrm{E}}$ a precipitação pluviométrica e a evaporação no estuário; $\mathrm{Q}_{\mathrm{as}} \mathrm{O}$ fluxo de água da drenagem subterrânea; $\mathrm{Q}_{\mathrm{r}}$ a vazão regularizada do reservatório; $\mathrm{Q}_{c}$ a contribuição de água do mar e $Q_{0}$ o fluxo de água resultante de oscilações discretas da maré. Todos os fluxos foram expressos $\mathrm{em} \mathrm{m}^{3} . \mathrm{s}^{-1}$ com sinais de positivo e negativo para as entradas e saídas de água no sistema hídrico, respectivamente.

As vazões das sub-bacias afluentes ao açude Catú-Cinzento construído em 2002 foram utilizadas na simulação do aporte de águas continentais no sistema estuarino antes do referido barramento.

\section{RESULTADOS E DISCUSSÕES}

Contribuições Hidráulicas e Balanço de Água no Sistema Estuarino

As médias históricas demonstram que a precipitação pluviométrica anual incidente sobre as cabeceiras e bacia estuarina são de aproximadamente de 1.042 e $1.456 \mathrm{~mm}\left(4,02.10^{-7}\right.$ e $\left.5,66.10^{-7} \mathrm{~m} \cdot \mathrm{s}^{-1}\right)$. No período de monitoramento (2003-2004), os totais preci-

TABELA 2: Variação do coeficiente de escoamento superficial e da descarga fluvial de cada sub-bacia $\left(\mathrm{m}^{3} \mathrm{~s}^{-1}\right)$

\begin{tabular}{|c|c|c|c|c|c|c|c|c|c|c|c|c|c|c|c|c|c|}
\hline \multirow[t]{2}{*}{ Bacias } & \multicolumn{2}{|c|}{ SB1 * } & \multicolumn{2}{|c|}{$\mathrm{SB} 2 * *$} & \multicolumn{2}{|c|}{ SB3** } & \multicolumn{2}{|c|}{ SB4** } & \multicolumn{2}{|c|}{ SB5** } & \multicolumn{2}{|c|}{ SB6** } & \multicolumn{2}{|c|}{ SB7** } & \multicolumn{2}{|c|}{$\mathrm{SB} 8 * *$} & \multirow[b]{2}{*}{ Total } \\
\hline & $\Delta \mathrm{f} / \mathrm{P}$ & $q_{f i}$ & $\Delta \mathrm{f} / \mathrm{P}$ & $q_{f i}$ & $\Delta \mathrm{f} / \mathrm{P}$ & $q_{f i}$ & $\Delta \mathrm{f} / \mathrm{P}$ & $q_{f i}$ & $\Delta \mathrm{f} / \mathrm{P}$ & $q_{\mathrm{fi}}$ & $\Delta \mathrm{f} / \mathrm{P}$ & $q_{f i}$ & $\Delta \mathrm{f} / \mathrm{P}$ & $q_{\mathrm{fi}}$ & $\Delta \mathrm{f} / \mathrm{P}$ & $\mathrm{q}_{\mathrm{fi}}$ & \\
\hline Jan & 0,19 & 0,59 & 0,19 & 0,18 & 0,19 & 0,13 & 0,15 & 0,04 & 0,15 & 0,05 & 0,15 & 0,04 & 0,15 & 0,02 & 0,15 & 0,14 & 1,19 \\
\hline Fev & 0,18 & 0,55 & 0,18 & 0,16 & 0,18 & 0,12 & 0,33 & 0,16 & 0,33 & 0,18 & 0,33 & 0,17 & 0,33 & 0,07 & 0,33 & 0,54 & 1,95 \\
\hline M ar & 0,44 & 2,71 & 0,44 & 0,80 & 0,44 & 0,61 & 0,54 & 0,45 & 0,54 & 0,51 & 0,54 & 0,49 & 0,54 & 0,19 & 0,54 & 1,50 & 7,25 \\
\hline Abr & 0,47 & 3,05 & 0,47 & 0,90 & 0,47 & 0,69 & 0,56 & 0,50 & 0,56 & 0,58 & 0,56 & 0,55 & 0,56 & 0,21 & 0,56 & 1,69 & 8,18 \\
\hline M ai & 0,27 & 1,01 & 0,27 & 0,30 & 0,27 & 0,23 & 0,35 & 0,17 & 0,35 & 0,20 & 0,35 & 0,19 & 0,35 & 0,07 & 0,35 & 0,58 & 2,76 \\
\hline Jun & 0,09 & 0,19 & 0,09 & 0,06 & 0,09 & 0,04 & 0,30 & 0,13 & 0,30 & 0,15 & 0,30 & 0,14 & 0,30 & 0,05 & 0,30 & 0,43 & 1,20 \\
\hline Jul & 0,00 & 0,00 & 0,00 & 0,00 & 0,00 & 0,00 & 0,07 & 0,01 & 0,07 & 0,01 & 0,07 & 0,01 & 0,07 & 0,01 & 0,07 & 0,04 & 0,09 \\
\hline Ago & 0,00 & 0,00 & 0,00 & 0,00 & 0,00 & 0,00 & 0,00 & 0,00 & 0,00 & 0,00 & 0,00 & 0,00 & 0,00 & 0,00 & 0,00 & 0,00 & 0,00 \\
\hline Set & 0,00 & 0,00 & 0,00 & 0,00 & 0,00 & 0,00 & 0,00 & 0,00 & 0,00 & 0,00 & 0,00 & 0,00 & 0,00 & 0,00 & 0,00 & 0,00 & 0,00 \\
\hline Out & 0,00 & 0,00 & 0,00 & 0,00 & 0,00 & 0,00 & 0,00 & 0,00 & 0,00 & 0,00 & 0,00 & 0,00 & 0,00 & 0,00 & 0,00 & 0,00 & 0,00 \\
\hline Nov & 0,00 & 0,00 & 0,00 & 0,00 & 0,00 & 0,00 & 0,00 & 0,00 & 0,00 & 0,00 & 0,00 & 0,00 & 0,00 & 0,00 & 0,00 & 0,00 & 0,00 \\
\hline Dez & 0,00 & 0,00 & 0,00 & 0,00 & 0,00 & 0,00 & 0,00 & 0,00 & 0,00 & 0,00 & 0,00 & 0,00 & 0,00 & 0,00 & 0,00 & 0,00 & 0,00 \\
\hline Média & - & 0,68 & - & 0,20 & - & 0,15 & - & 0,12 & - & 0,14 & - & 0,13 & - & 0,05 & - & 0,41 & \\
\hline
\end{tabular}
com base nas médias históricas.

Fonte: Aplicação das equações 2,3 e 4. SB - Sub-Bacia / $\Delta \mathrm{f} / \mathrm{P}$ - Razão de Escoamento, Superficial / $\mathrm{q}_{\mathrm{fi}}$ - Descarga da Sub-Bacia.

* Sub-bacias afluentes ao açude Catú-Cinzento. ** sub-bacias afluentes a lagoa do Catú 
pitados foram 1.199 e $1.753 \mathrm{~mm}\left(4,63.10^{-07}\right.$ e $6,76.10^{-}$ $\left.{ }^{07} \mathrm{~m} . \mathrm{s}^{-1}\right)$ o que representou um aumento variando de 15 e $20,5 \%$ em relação a média histórica de chuvas para região. As taxas de evaporação são bastante elevadas com um total anual de $5,67 \cdot 10^{-7} \mathrm{~m} \cdot \mathrm{s}^{-1}$. As maiores e menores taxas foram de $6,7 \mathrm{E} \cdot 10^{-8} \mathrm{e}-2,6 \cdot 10^{-08} \mathrm{~m} \cdot \mathrm{s}^{-1} 1$, registradas nos meses de outubro e abril, respectivamente. A temperatura média da região é de $26,6^{\circ} \mathrm{C}$.

Para o período de monitoramento (2003-2004), quando os dados de pluviometria tiveram maior representatividade para cada sub-bacia, a tendência à deficiência hídrica se manteve (-1472 mm). No entanto, as diferenças entre as taxas de evaporação e precipitação foram menores do que as baseadas em séries históricas. Por isso, nos estudos de contribuição hídrica optou-se por utilizar os dados das séries históricas.

A avaliação das características hidrológicas mostra um sistema bastante marcado pelos efeitos da sazonalidade hidroclimática. Praticamente toda a água doce que poderia chegar ao sistema estuarino no decorrer de um ano, escoa durante quatro meses. As variações da descarga fluvial de cada sub-bacia afluente ao sistema estuarino estão apresentadas na TABELA 2, baseada nas séries de dados históricos.

A contribuição hidrológica da bacia para a La- goa do Catú, antes do barramento era de $5.10^{7} \mathrm{~m}^{3}$, restrita ao período de janeiro a junho. Após a construção do reservatório Catú-Cinzento, em 2002, a contribuição reduziu para $4.10^{6} \mathrm{~m}^{3}$ (FIGURA 4). Neste caso, a bacia 1 seria responsável por aproximadamente $36 \%$ da contribuição de água para o sistema estuarino. A bacia 8 , localizada na área de influência direta da lagoa é atualmente responsável por $34 \%$ da contribuição de água' doce para o sistema estuarino. Vale salientar, que essas estimativas não consideraram as chuvas de recorrência milenar.

Nos estudos realizados pela SRH (1995) foi estimada uma vazão milenar afluente a lagoa do Catú de $222 \mathrm{~m}^{3} \cdot \mathrm{s}^{-1}$. A sugestão do projeto foi a diminuição do volume acumulado de $18.10^{6} \mathrm{~m}^{3}$ para $10.10^{6} \mathrm{~m}^{3} \mathrm{com}$ cota média de $5,8 \mathrm{~m}$. O volume atual do reservatório é de $7,5 \cdot 10^{6} \mathrm{~m}^{3}$.

A construção do reservatório Catú-Cizento associado ao sistema de vertimento da lagoa foi imprescindível no controle das inundações. No entanto, apesar da eficiência das obras, as margens da lagoa foram largamente desmatadas e ocupadas, aumentando o assoreamento e o surgimento de novas áreas de riscos de inundações.

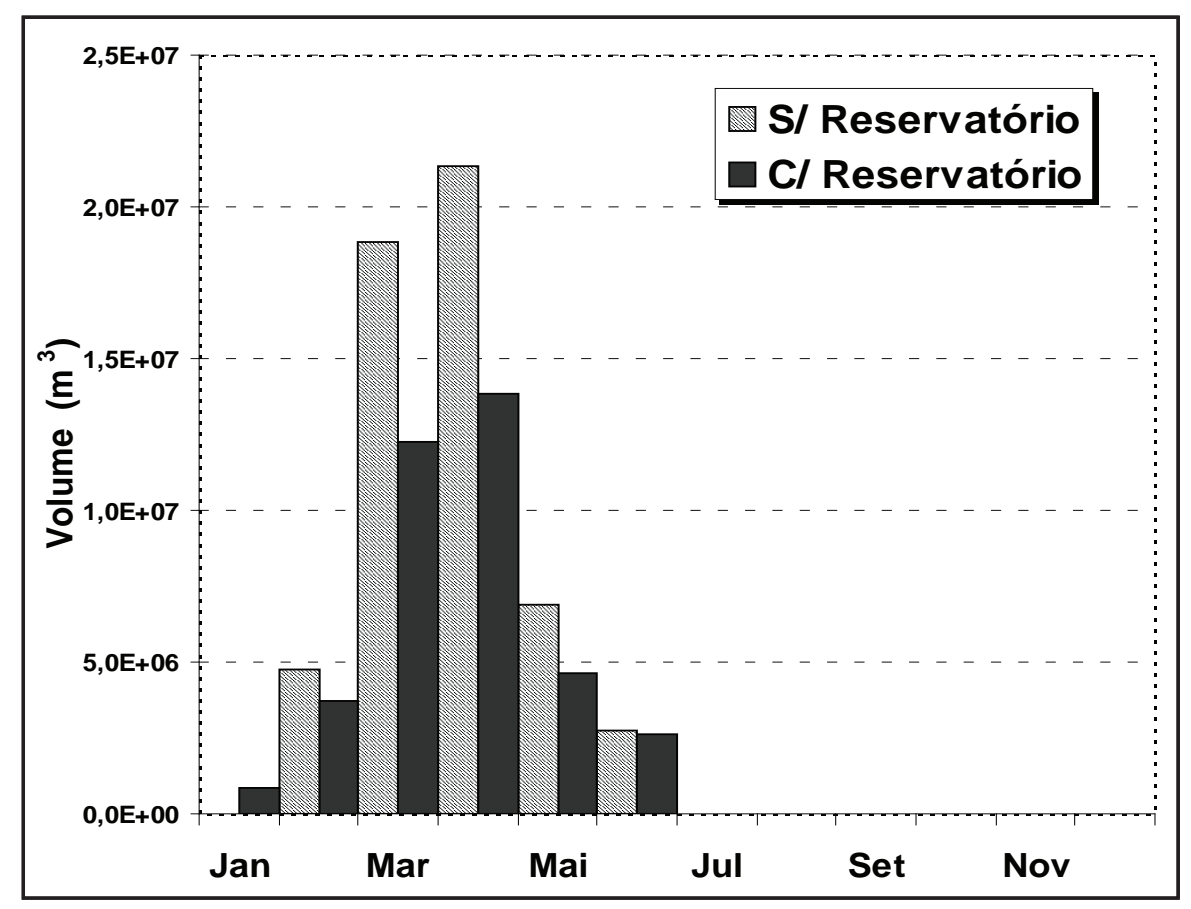

FIGURA 4: Volume de água afluente a Lagoa do Catú antes e após a construção do Açude Catú-Cinzento. 
As descargas fluviais das sub-bacias afluentes ao sistema estuarino são utilizadas como subsídio à elaboração do balanço total de água no sistema estuarino (PINHEIRO, 2003). Como pode ser observada na FIGURA 5, a intervenção da Prefeitura no Vertedouro natural da lagoa em 1993 (Caso 1) além de provocar a inundação das áreas ocupadas nas margens da lagoa do Catú, reduziu drasticamente a vazão de água para o sistema estuarino. A vazão anual afluente neste ano foi de aproximadamente $1,22 \mathrm{~m}^{3} \cdot \mathrm{s}^{-1}$.

Considerado o período de estiagem prolongado do ano anterior, a vazão não foi suficiente para promover a reabertura da foz, predominando neste caso os processos de assoreamento e morte do manguezal ali existente.

Com o objetivo de reverter os processos de inundação a montante e desaparecimento do estuário, a lâmina de vertimento da lagoa em 1995 foi reduzida em $1,51 \mathrm{~m}$. Isto promoveu o incremento na descarga fluvial para o total anual de $7,2 \mathrm{~m}^{3} \cdot \mathrm{s}^{-1}$, concentrados nos meses de março e abril (Caso 2).

Com a operação do reservatório Catú-Cinzento e vazão regularizada de $0,2 \mathrm{~m}^{3} \cdot \mathrm{s}^{-1}$, a contribuição anual para o estuário reduziu para $3,47 \mathrm{~m}^{3} \cdot \mathrm{s}^{-1}$. No período de estiagem a contribuição é nula e as altas taxas de evaporação propiciam a hipersalinização. No período de estiagem de 2003 e 2004, o substrato estuarino ficou exposto em mais de $70 \%$ da área.

A vazão liberada no período de estio não favorece os processos de mistura e circulação típicos de um ambiente estuarino e com repercussões na produtividade biológica e taxas de assoreamento na desembocadura.

As descargas fluviais das sub-bacias afluentes a lagoa do Catú foram utilizadas como subsídio à elaboração do balanço total de água no sistema estuarino. A contribuição da bacia de drenagem $\left(\mathrm{Q}_{\mathrm{f}}\right)$ para esse balanço foi obtida através da correção das vazões regularizadas e de sangria. Foi calculado o escoamento difuso incidente diretamente sobre o espelho d'água $\left(\mathrm{Q}_{\mathrm{d}-\mathrm{Q}_{\mathrm{evap}}}\right)$. Para os fluxos de água subterrânea $\left(Q_{a s}\right)$, devido a falta de dados disponíveis para o cálculo desse parâmetro, assumiu-se o valor igual a zero. Esse valor foi adotado com base no conhecimento da pouca permeabilidade da estrutura geológica da área e das altas taxas de evaporação características de regiões semi-áridas (PINHEIRO et al., 2006).

As descargas fluviais associadas à contribuição do vertedouro correspondem ao somatório entre as vazões, regularizadas e de sangria. Partindo do pres-

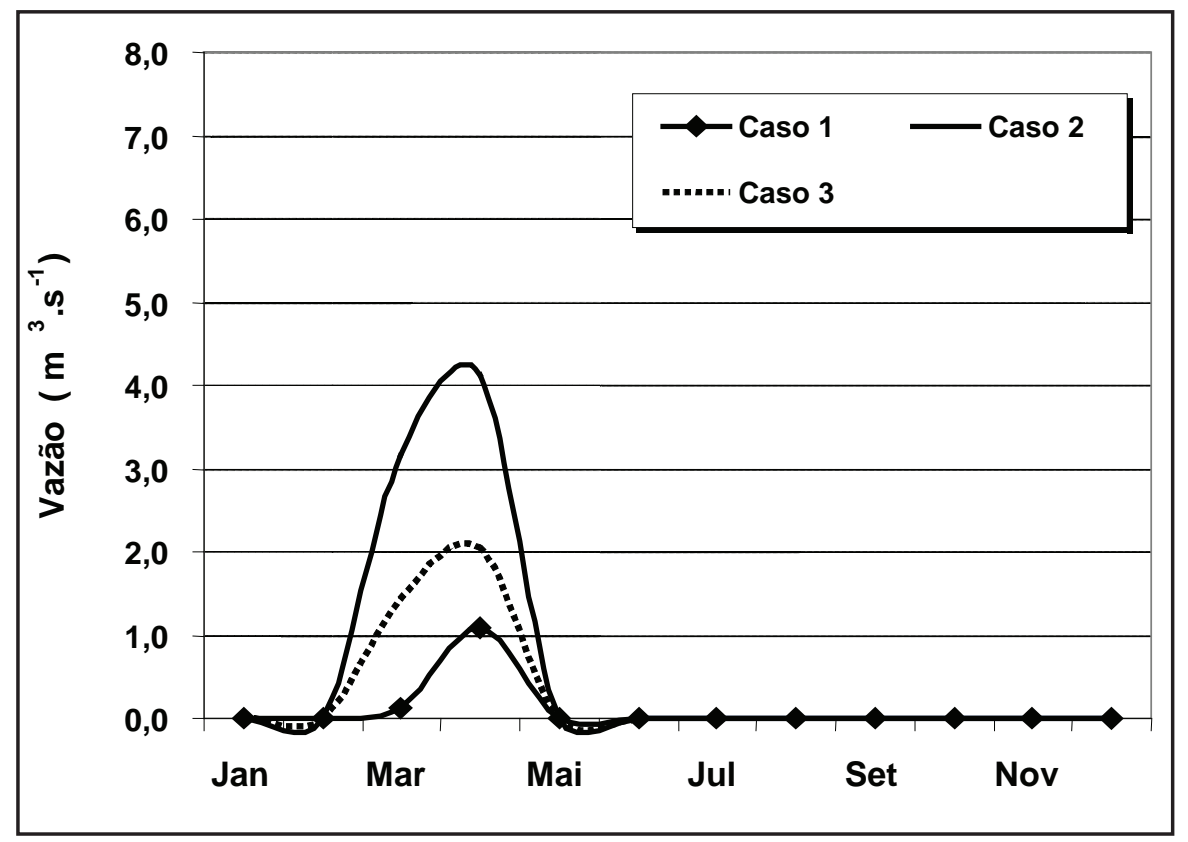

FIGURA 5: Evolução das vazões afluentes (Qf) ao estuário do Rio Catú. *Caso 1: Construção de vertedouro com barramento total em 1993; Caso 2: Rebaixamento da cota de vertimento em 1,51 m e Caso 3: Construção do Açude Catú-Cinzento. 
suposto de que o volume de água do estuário não varia ao longo dos anos, a contribuição resultante das oscilações da maré $\left(Q_{c}\right)$ foi obtida pela diferença necessária para satisfazer a seguinte condição:

$$
\frac{\Delta \mathrm{V}}{\Delta \mathrm{T}}=0
$$

Os valores positivos representam entrada de água e os negativos a perda.

O comportamento histórico demonstra nitidamente que as variações sazonais da descarga fluvial governam a hidrodinâmica do sistema estuarino. No período chuvoso foi identificado equilíbrio das massas de água, com uma pequena supremacia das mas-

\begin{tabular}{|c|c|c|}
\hline Período & Variáveis $\left(\mathrm{m}^{3} \mathrm{~s}^{-1}\right)$ & Estuário do Rio Catú \\
\hline \multirow{4}{*}{ Chuvoso } & $Q_{f}$ & 0,58 \\
\cline { 2 - 3 } & $Q_{d}$ & 0,01 \\
& $Q_{\text {evap }}$ & $-0,01$ \\
& $Q_{a s}$ & 0,00 \\
& $Q_{r}$ & 0,20 \\
& $Q_{c}$ & 0,79 \\
\hline \multirow{5}{*}{ Estiagem } & $Q_{f}$ & 0,00 \\
& $Q_{d}$ & 0,00 \\
& $Q_{\text {evap }}$ & $-0,01$ \\
& $Q_{a s}$ & 0,00 \\
& $Q_{r}$ & 0,20 \\
& $Q_{c}$ & 0,19 \\
\hline
\end{tabular}

QUADRO 1: Variáveis relacionadas ao balanço de água no estuário do Rio Catú-Cascavel-CE

Fonte: Aplicação da equação 8

sas de água salinas $(0,79)$ na composição do volume total do estuário que é de $8.507 \mathrm{~m}^{3}$

No período de estiagem, o mesmo equilíbrio foi observado com pequena supremacia de $0,01 \mathrm{~m}^{3} \cdot \mathrm{s}^{-1}$ das águas continentais. $\mathrm{O}$ volume médio para este período foi de $2.045 \mathrm{~m} 3$. Sob essas condições, a interação entre as massas d'água tende a formar suave gradiente de densidade que favorece os processos de mistura de natureza baroclínica. O tempo de residência das águas no sistema em um ciclo de maré é de 18 horas, aproximadamente. A capacidade de reciclagem das águas é muito baixa, que associada a baixa profundidade é um fator limitante para o uso intensivo das águas e emissão de quaisquer tipos de efluentes.

Durante o período de estiagem as oscilações da maré representam o principal mecanismo de entrada de água no sistema o que favorece os transportes advectivos e difusivos de natureza barotrópica (MIRANDA et al., 2002). Isto foi observado nos estuários do Rio Malcozinhado, Jaguaribe e Timonha por Pinheiro et al. (2006), Morais et al. (2006) e Dias (2005). No entanto, a descarga proveniente da vazão regulariza- da pelo reservatório, minimiza os efeitos do bombeamento gerado pela maré através da manutenção de uma forçante ligada ao escoamento de água doce que gera movimento estuário abaixo (DYER, 1997).

A posição geográfica ocupada pelo estuário do Rio Catú em função dos barramentos é o principal elemento de alta vulnerabilidade a degradação ambiental. Primeiro pela redução da área de contribuição hidráulica, alto poder de infiltração dos solos da área o que diminui o escoamento superficial, alta densidade de ocupação das áreas ribeirinhas e morfodinâmica dunar intensa. Além disso, a ausência de estudos sistemáticos que avaliassem os impactos da diminuição da descarga hídrica no sistema estuarino em curto, médio e longos prazos comprometeu de forma significativa a capacidade de suporte e a dinâmica natural deste sistema.

\section{CONCLUSÕES}

- O estuário do rio Catú é um ambiente estuarinolagunar em fase de evolução para ambiente lacustre; 
- A redução da descarga fluvial pelo superdimensionamento do vertedouro da Lagoa do Catú na década de 1990 representou o principal vetor de degradação ambiental e comprometimento da capacidade de suporte do estuário;

- A construção da barragem Catú-Cinzento em 2003, promoveu a diminuição em $36 \%$ da vazão de água doce ao estuário do Catú tornando-o susceptível aos processos de hipersalinização.

- O tempo de residência das águas no estuário é de aproximadamente 18 dias, o que confere uma baixa capacidade de suporte a emissão de efluentes.

\section{AGRADECIMENTOS}

A Secretaria de Recursos Hídricos do Estado do Ceará (SRH) e ao Banco Mundial pelo financiamento do projeto "Diagnóstico Geoambiental e Socioeconômico e proposta de monitoramento das áreas estuarinas e de manguezais dos Rios Malcozinhado, Catú, Timonha e Jaguaribe-PROGERHIR". A FUNCEME e COGERH pela disponibilidade dos dados. Ao $\mathrm{CNPq}$ pelas bolsas de produtividade em pesquisa dos autores. Aos revisores pelas valiosas discussões e contribuições.

\section{REFERÊNCIAS}

ALBER, M. A conceptual model of estuarine freshwater inflow management. Estuaries, 25(68), p. 12461261, 2002.

ARAÚJO, J. C. de; GÜNTNER, A.; BRONSTERT, A. Loss of reservoir volume by sediment deposition and its impact on water availability in semiarid Brazil. Hydrological Sciences-Journal-des Sciences Hydrologiques, 51(1), 2006.

BRANDT, S. A. Classification of Geomorphological effects downstream of Dams. Catena, 40(4), p. 375401, 2000.

CARVALHO, N. de O.; CUNHA, S. B. Estimativa de Carga Sólida do Rio Amazonas e seus principais Tributários para a Foz e Oceano: Uma Retrospectiva. A água em revista. Ano VI, n.6, p.44-58, 1998.
CAVAlCANTE, A. A. Aspectos da Produção de Sedimentos e seus Efeitos na Gestão dos Recursos no Baixo Vale do Rio Jaguaribe. 2001, 139 f. Dissertação (Mestrado em Geografia) - Universidade Estadual do Ceará, Ceará, 2001.

DIAS, C. B. Dinâmica do sistema estuarino Timonha/ Ubatuba (Ceará - Brasil) considerações ambientais. 2005. 180p. Dissertação (Mestrado em Ciências Marinhas Tropicais) - Universidade Federal do Ceará, 2005.

DOMINGUEZ, J. M. L.; BITTENCOURT, A. C. S. P.; MARTIN, L.O papel da deriva litorânea de sedimentos arenosos na construção das planícies costeiras associadas às desembocaduras dos rios São Francisco (SE/AL), Jequitinhonha (BA), Doce (ES) e Paraíba do Sul (RJ). Revista Brasileira de Geociências, São Paulo, v. 13, n. 4, p. 98-105, 1983.

DYER, K. R. Estuaries: a physical introduction. 2. ed. New York, John Wiley \& Sons, 1997.

FERNADEZ, O. V. Q; SOUZA FILHO, E. E. Efeitos do Regime Hidrológico sobre a evolução de um conjunto de Ilhas no rio Paraná. Boletim Paranaense de Geociências. Curitiba: Ed. da UFPR, 1995.

GENZ, F., LESSA, G. C.; CIRANO, M. Vazão mínima para estuários: um estudo de caso no rio Paraguaçu (BA). Revista Brasileira de Recursos Hídricos, v. 13, p. 73-82, 2008.

GOMES, M. L. Aspectos hidrológicos, sedimentológicos e impactos ambientais na lagoa costeira do Rio Catú-Aquiraz-Ceará. 2003. 138p. Dissertação (Mestrado em Geografia) - Universidade Estadual do Ceará, 2003.

KITHEKA, J. U.; OBIERO, M.; NTHENGE, P. River discharge, sediment transport and Exchange in the Tana Estuary, Kenya. Estuarine, Coastal and Shelf Science, 63, p. 455-468, 2005.

KJERFVE, B. Estuarine characteristics, circulation and physical processes. In: DAY JR, J.W.; et al. Es- 
tuarine Ecology. Jonh Wiley and Sons Inc, New York, NY, p.47-78, 1990.

KJERFVE, B. Coastal lagoon processes. New York, Elsevier, 1994.

KJERFVE, B.; SCHETTINI, C. A. F. C. A. F.; KNOPPERS, B.; LESSA, G. C.; FERREIRA, H. O. Hydrology and salt balance in the world's largest hypersaline coastal lagoon: Lagoa de Araruama, Brazil. Estuarine, Coastal and Shelf Science, Estados Unidos, v. 42, p. 701-725, 1996.

LATRUBESSE, E. M.; STEVAUX, J. C.; SINHÁ, R. Tropical Rivers. Geomorphology, 70, p. 187-206, 2005 .

LIMA, W. P.; ZAKIA, M. J. B. Indicadores hidrológicos. Série Técnica IPEF, v. 12, n. 31, p. 53-64, 1998.

MARINS, R. V.; LACERDA, L. D.; ABREU, I. M.; DIAS, F. J. S. Efeitos da açudagem no rio Jaguaribe. Ciência Hoje, Rio de Janeiro, v. 33, n. 197, p. 66-70, 2003.

MARTIN, L.; DOMINGUEZ, J. M. L. Geological history of coastal lagoons. In: KJERVE, B. Coastal Lagoon Processes. London: Elsevier Oceanography Series, p. 41-66, 1994.

MEDEIROS, C.; KJERFVE, B. Hydrology of a Tropical Estuarine System: Itamaracá, Brazil. Estuarine, Coastal and Shelf Science, 36(1), p. 436-515, 1993.

MIRANDA, L. B.; CASTRO, B. M.; KJERFVE, B. Princípios de Oceanografia Física de Estuários. EDUSP: Universidade de São Paulo, 2002.

MOLISANI, M. M.; CRUZ, A. L. V.; MAIA, L. P. Estimativas das descargas fluviais para os estuários do Estado do Ceará. Arquivos de Ciências do Mar, v.39, p. 53-60, 2006.

MOLISANI, M. M.; KJERFVE, B.; LACERDA, L. D. Water Discharge and Sediment Load to Sepetiba Bay from an Anthropogenically-Altered Drainage
Basin, SE Brazil. Journal of Hydrology, v. 331, p. 425-433, 2006.

MORAIS, J. O.; IRION, G. F. ; PINHEIRO, L. S. ; MEDEIROS, C. Aspectos sedimentológicos comparativos entre o sistema estuarino do Malcozinhado e Lagoa da Encantada-Ceará. Essentia (Sobral/CE), Sobral, v. 4, n. 1, p. 101-113, 2002.

MORAIS, J. O. de ; PINHEIRO, L. S. ; PAULA, D. P. de. Dinâmica ambiental do Estuário do Rio Jaguaribe: impactos nos processos termohalinos a partir da construção de barragens. In: PINHEIRO, D. R. C. (Org.). Desenvolvimento sustentável: desafios e discussões. 1 ed. Fortaleza: ABC, v. 1, p. 95-108, 2006.

PINHEIRO, L. S. Riscos e Impactos Ambientais no Estuário do Rio Malcozinhado, Cascavel-CE. 2003. 235p. Tese (Doutorado em Oceanografia) - Universidade Federal de Pernambuco, 2003.

PINHEIRO, L. S.; MEDEIROS, C.; MORAIS, J. O. Erosive processes monitoring linked to the estuarine evolution systems nearby Aguas Belas, Cascavel, Ceará, Brazil. Journal of Coastal Research, Itajaí, v. 39, n. 1, p. 1403-1406, 2006.

SKLAR F. H., BROWDER, J. A. Coastal Environmental Impacts Brought about by alterations to freshwater flow in the Gulf of Mexico. Environmental Management, 22(4), p. 547-562, 1998.

SRH-Secretaria dos Recursos Hídricos do Estado do Ceará. Barramentos da Lagoa do Catú. Relatório Interno. Acesso restrito, 1995.

STANFORD, J. A., WARD, J.V.; LISS, W. J.; FRISSEL, C. A.; WILLIAMS, R. N.; LICHATOWICH, J. A; COUTANT, C. C. A general protocol for restoration of regulated rivers. Regulated Rivers, Research and Managment, 12, p. 391-413, 1996.

TOWNSEND C.R.; HILDREW A.G. Species traits in relation to a habitat templet for river systems. Freshwater Biology, 31, p. 265-275, 1994. 
WOLANSKI, E.; HUAN, N. N.; DAO, L. T.; NHAN, N. H ; THUY, N. N. Fine-sediment dynamics in the Mekong River Estuary, Vietnam. Estuarine, Coastal and Shelf Science; 43, p. 565-582, 1996. 